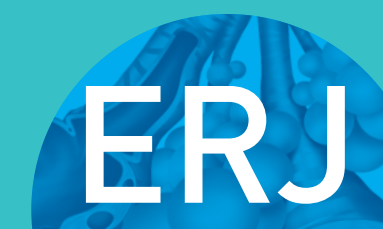

open research
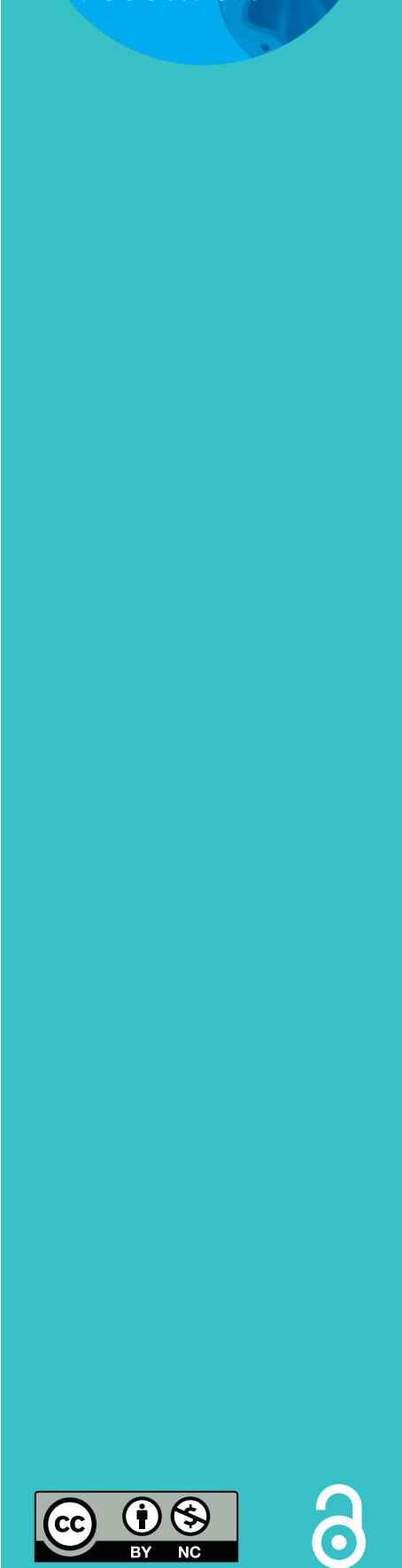

\section{Lower extremity and carotid artery disease in COPD}

\author{
Carmen Pizarro ${ }^{1,3}$, Fabian Linnhoff $^{1,3}$, Fabian van Essen ${ }^{1}$, Simon Pingel ${ }^{1}$, \\ Christian Alexander Schaefer ${ }^{1}$, Nadjib Schahab ${ }^{1}$, Rolf Fimmers ${ }^{2}$, \\ Georg Nickenig ${ }^{1}$ and Dirk Skowasch ${ }^{1}$
}

Affiliations: 'Dept of Internal Medicine II, Cardiology, Pneumology and Angiology, University Hospital Bonn, Bonn, Germany. ${ }^{2}$ Institute for Medical Biometry, Informatics and Epidemiology, University Hospital Bonn, Bonn, Germany. ${ }^{3}$ These authors contributed equally to this work.

Correspondence: Carmen Pizarro, Dept of Internal Medicine II, Cardiology, Pneumology and Angiology, University Hospital Bonn, Sigmund-Freud-Straße 25, 53105 Bonn, Germany.

E-mail: carmen.pizarroQukb.uni-bonn.de

ABSTRACT In view of their common chronic inflammatory process, we sought to determine the linkage between peripheral artery disease and chronic obstructive pulmonary disease (COPD).

107 COPD patients (mean \pm SD age $64.6 \pm 10.4$ years, $52.2 \%$ male) and 22 control smokers without previously diagnosed peripheral artery disease underwent standardised angiological examination for lower extremity artery disease (LEAD) and carotid artery disease.

LEAD was significantly more prevalent in COPD patients than in controls $(80.4 \%$ versus $54.5 \%$, $\mathrm{p}=0.002$ ). Among COPD patients, $57.0 \%, 12.2 \%, 10.3 \%$ and $0.9 \%$ were found to be in Fontaine stages I, IIA, IIB and III, respectively. As with carotid artery disease, its frequency increased from $36.4 \%$ in controls to $58.9 \%$ in COPD patients $(\mathrm{p}=0.003$ ). Carotid plaque burden, LEAD Fontaine degrees as well as pulse wave index and ankle-brachial index manifested significant impairment over percentage predicted forced expiratory volume in $1 \mathrm{~s}$ (FEV1 \% pred) $(\mathrm{p}=0.02, \mathrm{p}<0.001, \mathrm{p}=0.01$ and $\mathrm{p}<0.001$, respectively). Multivariate analysis revealed that COPD Global Initiative for Chronic Obstructive Lung Disease status was the strongest independent predictor for the presence of plaque in lower extremity arteries (odds ratio 1.63, 95\% CI 1.19-2.25, $\mathrm{p}=0.003$ ) and carotids (odds ratio 1.66, 95\% CI 1.14-2.44, $\mathrm{p}=0.009$ ).

As compared with control smokers, peripheral artery disease is diagnosed in a sizeable proportion of COPD patients and exhibits significant distributive differences over FEV1 \% pred that exceed the susceptibility conferred by common cardiovascular stressors.

@ERSpublications

Undiagnosed lower extremity and carotid artery disease is common in COPD and aggravates over COPD GOLD stages http://ow.ly/w96f303lHTu

Received: Jan 132016 | Accepted after revision: Aug 122016

Conflict of interest: None declared.

Copyright $\odot$ ERS 2016. This article is open access and distributed under the terms of the Creative Commons Attribution Non-Commercial Licence 4.0. 


\section{Introduction}

Chronic obstructive pulmonary disease (COPD) constitutes a progressive, debilitating disease of increasing prevalence that causes substantial morbidity and mortality worldwide [1]. Albeit its diagnostic and therapeutic management are primarily guided by the severity of airflow limitation, the complexity of the disease course is importantly influenced by its extrapulmonary comorbid manifestations [2-4]. It is noteworthy that comorbidities considerably affect outcomes in COPD, with cardiovascular diseases constituting a major cause of death [5]. In COPD as a multicomponent disorder, the inflammatory response that results from exposure to noxious environmental fumes becomes noticeable at both the local and systemic level [6]. Atherosclerotic conditions may arise as a consequence of common risk factors, primarily tobacco smoke, and of a systemic pro-inflammatory state. As such, the onset and progress of peripheral artery disease is markedly influenced by vascular inflammatory processes [7]. Although numerous inflammatory variables have been identified to play a major role in both peripheral artery disease and COPD, interdependence and coexistence of both disease entities are conceivable but still undefined. In a study cohort comprising 30 patients with advanced peripheral artery disease, WOZNIAK et al. [8] spirometrically ascertained concomitant COPD to be present in $46.7 \%$ of patients. This observation hints at a mutual association and raises the question whether COPD vice versa impacts peripheral artery disease occurrence and progression. In keeping with this, we conducted this prospective, cross-sectional study to investigate the frequency of lower extremity artery disease (LEAD) and carotid artery disease in COPD patients and current or former control smokers, and related angiological results to severity of obstructive airflow limitation.

\section{Materials and methods}

Patient population

Between June 2014 and December 2015, a total of 107 patients aged $\geqslant 18$ years receiving treatment at the Dept of Pneumology, University Hospital Bonn (Bonn, Germany) for spirometrically and clinically confirmed COPD participated in this prospectively conducted study. All patients received outpatient care; none were locally hospitalised. Exclusion criteria for study participation comprised previously diagnosed LEAD and carotid artery disease (including cerebrovascular disorders) as well as acute exacerbated COPD at the time of evaluation. All patients underwent angiological examination and complementary laboratory testing. Comorbidities, established risk factors for cardiovascular disorders, smoking history and current medication use were assessed by standardised questionnaire and appraisal of medical reports (physician's letters, laboratory values, list of medication).

The control cohort consisted of ex-smokers or current smokers who were recruited from the general population by screening invitation and in whom no COPD or atherosclerotic disorder had been diagnosed beforehand. Potential controls underwent pulmonary function testing to confirm absence of COPD. 22 controls were matched against COPD patients for age, gender, smoking habits and frequency of concomitant cardiovascular risk factors, and likewise underwent the aforementioned diagnostics.

The study was approved by the Ethics Committee of the University of Bonn and complied with the Declaration of Helsinki. All patients gave their written informed consent prior to entry into the study.

\section{Pulmonary function testing}

In conformity with the current guidelines [1], classification of COPD severity was performed by post-bronchodilator spirometry assessment of airflow limitation, symptomatic evaluation using the validated COPD Assessment Test and appraisal of exacerbation history. Concurrently, body plethysmography, diffusion capacity testing for carbon monoxide and capillary blood gas analysis permitted quantification of hyperinflation, ventilation and oxygenation status.

\section{Laboratory testing}

All study participants underwent blood sampling that encompassed a complete blood cell count and high-sensitivity C-reactive protein (hs-CRP).

\section{Angiological screening}

Angiological testing comprised examination for both LEAD and carotid artery disease, and was performed by experienced angiologists, blinded to all clinical data, in line with the current European Society of Cardiology (ESC) guidelines [9]. For evaluation of carotid artery disease and quantification of its severity, colour-coded duplex ultrasonography was employed, according to the revised DEGUM (Ultraschall-Akademie der Deutschen Gesellschaft für Ultraschall in der Medizin) ultrasound criteria [10].

Noninvasive vascular testing for LEAD included ankle-brachial index (ABI) measurement, colour-coded duplex sonography as well as assessment of central pulse wave velocity (cPWV) and pulse wave index (PWI). Clinical LEAD categorisation accorded with Fontaine classification, and was ascertained by the 
treadmill test and anamnestic specification. Consistent with the current ESC guidelines on peripheral artery disease [9], ABI was calculated by Doppler-guided measurement of each foot's dorsalis pedis and posterior tibial arteries systolic pressure and its division by the systolic brachial pressure. Mean ABI, obtained by averaging the resulting four ABI values, was employed for statistical analysis. An ABI $<0.9$ indicated occlusive arterial disease. Complementary colour-coded duplex ultrasonography provided additional information as it allowed for visualisation of the vascular lesion's extent and severity, and consequently of the atherosclerotic plaque burden. As one of the major indicators for arterial stiffness, brachial-ankle cPWV was assessed [11]. PWI measurement permitted ascertainment of subclinical LEAD stages even in the absence of ABI alterations; a PWI $>180$ was considered pathological [12]. cPWV and PWI assessments were performed by use of an AngE Pro8 pulse oscillography device (Sonotechnik Austria, Maria Rain, Austria).

\section{Statistical analysis}

Categorical data were presented as absolute number and percentages; continuous variables were expressed as mean $\pm \mathrm{SD}$ or median (interquartile range). Comparison of continuous variables was carried out by the $t$-test or univariate ANOVA. For categorical parameters, Fisher's exact test or Pearson's Chi-squared test was employed. Correlation was assessed by use of Pearson's correlation coefficient. In order to describe the influence potential confounders have on lower extremity plaque presence as a binary dependent variable, a logistic regression model was employed. All statistical analyses were performed with SPSS Statistics version 23.0 (IBM, Armonk, NY, USA).

\section{Results}

Characteristics of the study population

Demographic features and clinical data of study participants are displayed in table 1. Intercohortal comparison was performed by defining controls as the COPD Global Initiative for Chronic Obstructive Lung Disease (GOLD) 0 group.

Within the COPD cohort, $5.6 \%, 48.6 \%, 3.7 \%$ and $42.1 \%$ of patients were in COPD GOLD groups A, B, C and $\mathrm{D}$, respectively. Pulmonary function testing revealed an overall forced expiratory volume in $1 \mathrm{~s}\left(\mathrm{FEV}_{1}\right) /$ forced vital capacity (FVC) ratio of $59.7 \pm 15.3 \%$ (table 2 ). FEV1 accounted for $1.6 \pm 0.8 \mathrm{~L}$ in absolute terms and $56.7 \pm 21.8 \%$ of the predicted value. Prior or continued nicotine consumption was exhibited by $95.3 \%$ of patients (median 30 pack-years); six out of 107 patients were $\alpha_{1}$-antitrypsin deficient. Capillary blood gas analysis showed a resting oxygen and carbon dioxide tension of $66.2 \pm 12.6$ and $36.2 \pm 6.8 \mathrm{mmHg}$, respectively. $19.6 \%$ of the COPD cohort was under long-term supplemental oxygen therapy. Bronchodilative and anti-inflammatory background medication in use at the time of study is given in table 1; eight out of 107 COPD patients had previously undergone endoscopic lung volume reduction (three and five patients by endobronchial valve and coil placement, respectively). With regard to cardiovascular medication, the use of antiplatelet agents and $\beta$-blockers significantly differed between groups, and correlated with the presence of coronary heart disease (Pearson's $\mathrm{r}=0.48, \mathrm{p}<0.001$ and Pearson's $\mathrm{r}=0.20, \mathrm{p}=0.03$, respectively). As for established cardiovascular risk factors, no significant distributive intercohortal differences were detected.

Among current or former control smokers, pulmonary function testing excluded presence of COPD: $\mathrm{FEV} 1 / \mathrm{FVC}$ ratio was $79.7 \pm 6.9 \%$ and $\mathrm{FEV} 1$ was $98.2 \pm 14.3 \%$ of predicted, although quantitative smoking history was balanced between controls and COPD patients $(\mathrm{p}=0.54)$.

\section{Angiological results}

Angiological testing identified LEAD to be present in 86 out of 107 patients $(80.4 \%$ of the COPD study population): $57.0 \%$ were found to be in Fontaine stage I, $12.2 \%$ in Fontaine stage IIA, $10.3 \%$ in Fontaine stage IIB and $0.9 \%$ in Fontaine stage III. Among controls, 12 out of 22 subjects (i.e. $54.5 \%$ of the control cohort) were in Fontaine stage I, whereas no higher LEAD grade was ascertained. Thus, as compared with controls, LEAD frequency was significantly higher among COPD patients $(80.4 \%$ versus $54.5 \%, \mathrm{p}=0.002)$. In order to evaluate the association between GOLD severity and LEAD Fontaine stages, univariate analysis demonstrated a significant increase of LEAD stages over GOLD classes $(p<0.001)$. Statistical significance was persistently upheld after combination of GOLD stages A and B as well as stages $\mathrm{C}$ and D into two categorical variables, given the limited number of patients in the less symptomatic GOLD stages A and C $(\mathrm{p}=0.003$ ) (figure 1). Vice versa, FEV1 decreased significantly over LEAD stages $(\mathrm{p}<0.001)$ (figure 2 ).

Pulse volume recording ascertained bilaterally concordant PWI values (Pearson's $\mathrm{r}=0.75, \mathrm{p}<0.001$ ); PWI was impaired significantly within COPD severity groups $(\mathrm{p}=0.007)$. $\mathrm{cPWV}$ was $7.21 \pm 3.62 \mathrm{~m} \cdot \mathrm{s}^{-1}$ and did not manifest significant distributive changes neither over COPD stages nor by comparison of COPD patients with controls. 
TABLE 1 Demographic and clinical characteristics of the study population

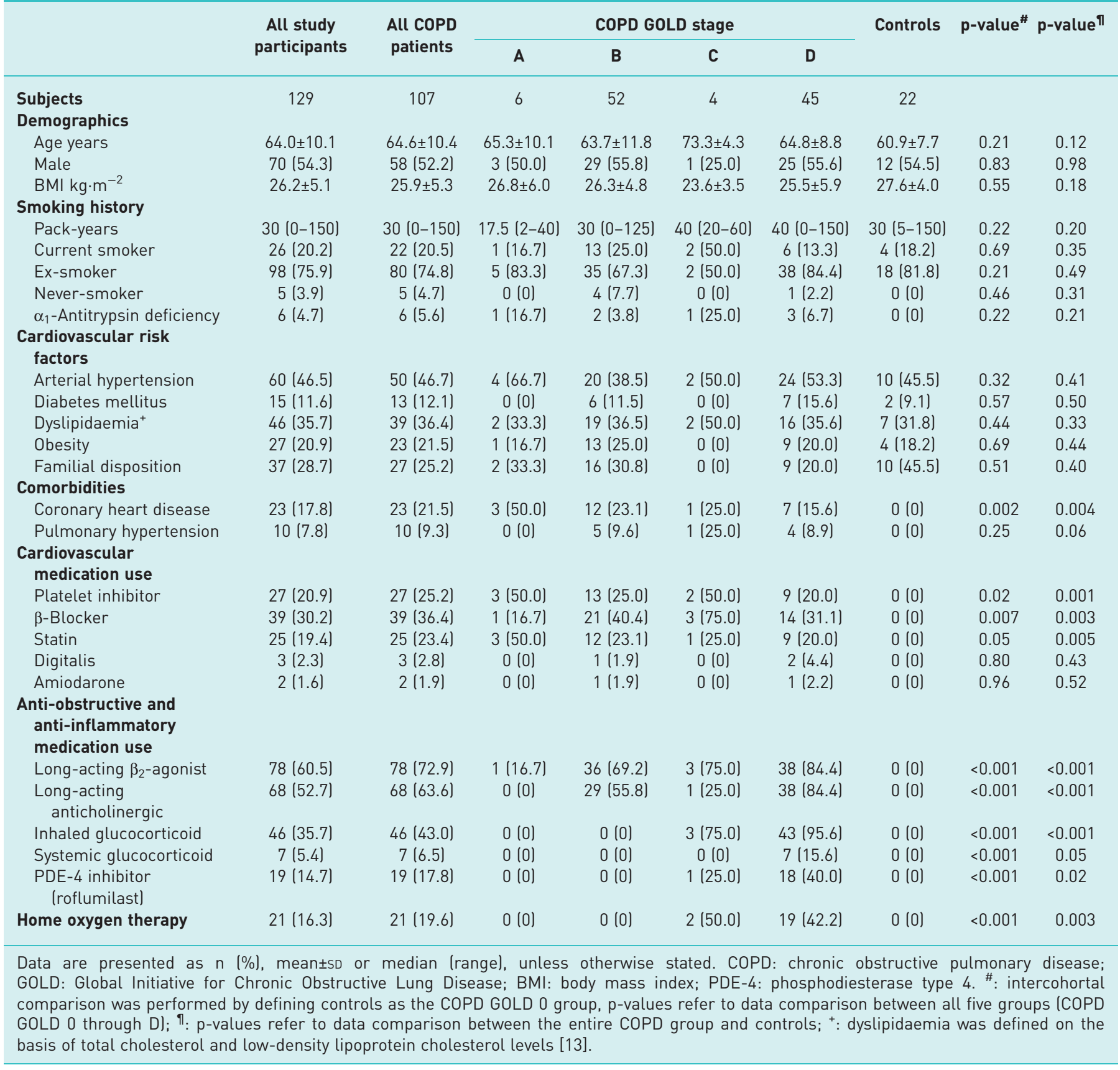

Although controls were not free from carotid artery atherosclerosis, as $36.4 \%$ had nonstenotic plaque in the common or internal carotid arteries, the frequency of carotid artery disease increased to $58.9 \%$ in COPD patients $(\mathrm{p}=0.003)$ and was accompanied by a significant increase in plaque load over COPD GOLD stages $(\mathrm{p}=0.04)$.

In the entire study population $(n=129)$, statistically significant relations were observed on linear regression analysis between COPD GOLD groups and $\mathrm{FEV}_{1} \%$ pred, on the one hand, and presence of plaque and stenosis in lower extremity arteries, plaque burden in carotid arteries, PWI, ABI, and leukocyte count, on the other hand (table 3 and figure 3). Concurrently, leukocyte count was significantly related to hs-CRP (Pearson's $\mathrm{r}=0.25, \mathrm{p}=0.01$ ) and pulmonary testing parameters indicating obstructive airflow limitation (FEV1 \% pred: Pearson's $\mathrm{r}=-0.31, \mathrm{p}=0.001$; FEV1/FVC ratio: Pearson's $\mathrm{r}=-0.23, \mathrm{p}=0.01$ ).

A stepwise multiple logistic regression analysis was used to further investigate the association between presence of plaque in lower extremity arteries and established LEAD risk factors (age, gender, pack-years 
TABLE 2 Pulmonary functional characterisation of the study population

\begin{tabular}{|c|c|c|c|c|c|c|c|c|c|}
\hline & $\begin{array}{c}\text { All study } \\
\text { participants }\end{array}$ & $\begin{array}{l}\text { All COPD } \\
\text { patients }\end{array}$ & \multicolumn{4}{|c|}{ COPD GOLD stage } & Controls & p-value ${ }^{\#}$ & p-value \\
\hline Subjects & 129 & 107 & 6 & 52 & 4 & 45 & 22 & & \\
\hline \multicolumn{10}{|l|}{$\begin{array}{l}\text { Pulmonary function } \\
\text { parameters }\end{array}$} \\
\hline FEV $1 \mathrm{~L}^{+}$ & $1.80 \pm 0.93$ & $1.57 \pm 0.77$ & $1.76 \pm 0.86$ & $2.01 \pm 0.74$ & $1.16 \pm 0.20$ & $1.26 \pm 0.37$ & $3.02 \pm 0.75$ & $<0.001$ & $<0.001$ \\
\hline $\mathrm{FEV}_{1} \%$ pred $^{+}$ & $63.7 \pm 25.6$ & $56.7 \pm 21.8$ & $70.3 \pm 15.0$ & $70.6 \pm 14.2$ & $48.1 \pm 2.5$ & $45.2 \pm 14.1$ & $98.2 \pm 14.3$ & $<0.001$ & $<0.001$ \\
\hline $\mathrm{FEV}_{1} / \mathrm{FVC}$ ratio ${ }^{+}$ & $62.9 \pm 15.9$ & $59.7 \pm 15.3$ & $57.5 \pm 10.9$ & $65.6 \pm 10.4$ & $56.9 \pm 18.1$ & $48.7 \pm 13.5$ & $79.7 \pm 6.9$ & $<0.001$ & $<0.001$ \\
\hline RV L & $3.68 \pm 1.57$ & $3.92 \pm 1.57$ & $3.76 \pm 1.40$ & $2.97 \pm 0.87$ & $3.58 \pm 1.20$ & $4.28 \pm 1.15$ & $2.51 \pm 0.91$ & $<0.001$ & $<0.001$ \\
\hline RV $\%$ pred & $163.9 \pm 68.5$ & $174.8 \pm 69.2$ & $166.1 \pm 50.4$ & $132.8 \pm 40.7$ & $141.7 \pm 23.5$ & $182.7 \pm 39.9$ & $111.3 \pm 34.8$ & $<0.001$ & $<0.001$ \\
\hline Rтот kPa.s. $\mathrm{L}^{-1}$ & $0.43 \pm 0.28$ & $0.47 \pm 0.29$ & $0.34 \pm 0.14$ & $0.34 \pm 0.18$ & $0.46 \pm 0.08$ & $0.63 \pm 0.33$ & $0.23 \pm 0.10$ & $<0.001$ & $<0.001$ \\
\hline Rтот \% pred & $143.1 \pm 95.2$ & $156.9 \pm 98.2$ & $113.9 \pm 47.9$ & $113.5 \pm 60.8$ & $153.6 \pm 27.3$ & $213.3 \pm 113.9$ & $77.2 \pm 32.6$ & $<0.001$ & $<0.001$ \\
\hline DLco $\%$ pred & $55.8 \pm 19.6$ & $48.5 \pm 16.0$ & $74.9 \pm 8.2$ & $53.9 \pm 11.4$ & $47.7 \pm 15.3$ & $36.3 \pm 15.2$ & $77.1 \pm 13.4$ & $<0.001$ & $<0.001$ \\
\hline $\mathrm{PCO}_{2} \mathrm{mmHg}$ & $36.0 \pm 6.5$ & $36.2 \pm 6.8$ & $33.5 \pm 6.2$ & $34.4 \pm 7.3$ & $37.4 \pm 0.8$ & $38.1 \pm 4.8$ & $35.0 \pm 4.6$ & 0.16 & 0.42 \\
\hline $\mathrm{SO}_{2} \%$ & $94.0 \pm 2.9$ & $93.6 \pm 3.0$ & $95.7 \pm 0.7$ & $93.9 \pm 3.3$ & $94.0 \pm 2.0$ & $91.8 \pm 2.9$ & $96.0 \pm 1.2$ & $<0.001$ & $<0.001$ \\
\hline \multicolumn{10}{|c|}{ 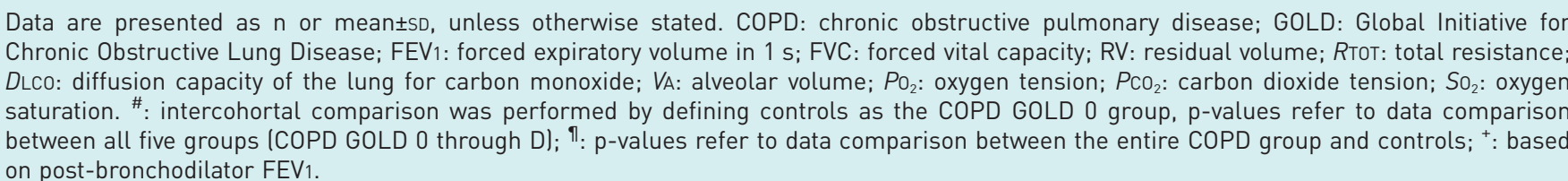 } \\
\hline
\end{tabular}

of smoking, arterial hypertension, diabetes mellitus, hyperlipidaemia and familial disposition). The only cardiovascular risk factor identified to be independently associated with plaque presence was age (odds ratio (OR) 1.05, 95\% CI 1.00-1.10, p=0.039). Overall, COPD GOLD status was the strongest independent predictor for presence of plaque both in lower extremity arteries (OR 1.63, 95\% CI 1.19-2.25, p=0.003) and carotids (OR 1.66, 95\% CI 1.14-2.44, $\mathrm{p}=0.009$ ).

\section{Discussion}

The present study conducted on a heterogeneous COPD population of considerable size demonstrates an unexpectedly high LEAD frequency of $80.4 \%$ that substantially exceeds the frequency ascertained in our matched control smokers without COPD. Both LEAD severity degrees and PWI manifested a significant increase with the degree of obstructive airflow limitation, whereas ABI values decreased. In terms of carotid artery disease, carotid plaque burden exhibited significant distributive differences over FEV1 \% pred.

FIGURE 1 Distribution of lower extremity artery disease (LEAD) Fontaine stages over the combined chronic obstructive pulmonary disease (COPD) Global Initiative for Chronic Obstructive Lung Disease (GOLD) groups $A / B$ and $C / D$ $(p=0.003)$.

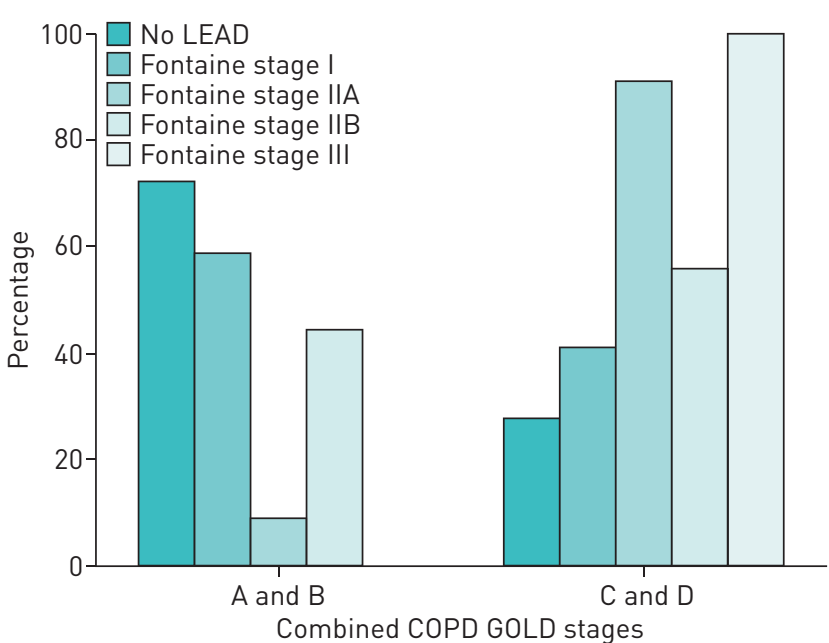


FIGURE 2 Forced expiratory volume in $1 \mathrm{~s}\left(\mathrm{FEV}_{1}\right) \%$ pred stratified by lower extremity artery disease (LEAD) Fontaine stages ( $p<0.001)$.

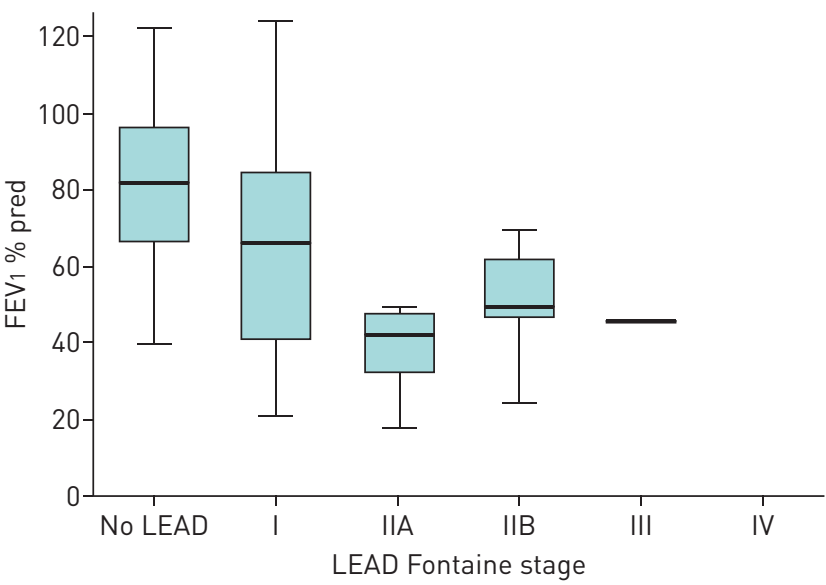

As a marker of systemic inflammation, total leukocyte count was related to both COPD and LEAD severity degrees.

Although the traditional diagnostic and therapeutic focus of COPD had rested on the presence and management of chronic airflow limitation, COPD has now been recognised as a systemic disorder whose course is substantially influenced by its comorbid conditions [4]. According to mortality statistics and large-scale clinical COPD trials $[14,15], 25-50 \%$ of mortality is attributable to cardiovascular comorbidities that thereby constitute a major cause of death in COPD. It is conceivable that the elevated cardiovascular morbidity and mortality arise from the common stressors, such as smoking and arterial hypertension, that COPD patients are subjected to. However, a growing body of evidence has associated reduced FEV1 per se with cardiovascular risk, irrespective of conventional cardiovascular risk stratifiers [16]. Whereas the concomitance of coronary heart disease and COPD has repeatedly been the focus of research $[17,18]$, the prevalence and mutual interaction of peripheral vascular disease in COPD remain less well defined. In a cohort of 30 patients with advanced LEAD according to at least Fontaine stage IIB, spirometry identified concomitant COPD in 14 cases [8]. Vice versa, in a Spanish cross-sectional COPD study, PECCI et al. [19] ascertained abnormal ABI values in $36.8 \%$ of COPD patients, of whom approximately $70 \%$ showed asymptomatic LEAD. Likewise, cross-sectional evidence that LEAD did not impaired over COPD severity stages was provided by WATz et al. [20]. Contrary to our results and broadly

TABLE 3 Correlation analysis between pulmonary, angiological and laboratory parameters in the study population

\begin{tabular}{|c|c|c|c|c|c|c|}
\hline & \multicolumn{2}{|c|}{ COPD GOLD stage } & \multicolumn{2}{|c|}{ FEV $1 \%$ pred } & \multicolumn{2}{|c|}{ RV \% pred } \\
\hline & Pearson's r & p-value & Pearson's r & p-value & Pearson's r & p-value \\
\hline \multicolumn{7}{|c|}{ Lower extremity artery disease } \\
\hline Fontaine stage & 0.413 & $<0.001$ & -0.433 & $<0.001$ & 0.230 & 0.02 \\
\hline Plaque burden & 0.373 & $<0.001$ & -0.371 & $<0.001$ & 0.182 & 0.05 \\
\hline Stenosis & 0.291 & 0.002 & -0.326 & $<0.001$ & 0.230 & 0.01 \\
\hline $\mathrm{ABI}$ (mean) & -0.385 & $<0.001$ & 0.386 & $<0.001$ & -0.224 & 0.01 \\
\hline PWI (mean) & 0.258 & 0.007 & -0.249 & 0.01 & 0.091 & 0.357 \\
\hline cPWV & 0.135 & 0.211 & -0.162 & 0.13 & 0.120 & 0.27 \\
\hline \multicolumn{7}{|l|}{ Carotid artery disease } \\
\hline Plaque burden & 0.184 & 0.04 & -0.216 & 0.02 & 0.040 & 0.68 \\
\hline \multicolumn{7}{|l|}{ Laboratory parameters } \\
\hline Total leukocyte count & 0.242 & 0.008 & -0.312 & 0.001 & 0.180 & 0.05 \\
\hline hs-CRP & 0.033 & 0.73 & -0.014 & 0.89 & 0.051 & 0.60 \\
\hline \multicolumn{7}{|c|}{$\begin{array}{l}\text { COPD: chronic obstructive pulmonary disease; GOLD: Global Initiative for Chronic Obstructive Lung } \\
\text { Disease; FEV1: forced expiratory volume in } 1 \mathrm{~s} \text {; RV: residual volume; ABI: ankle-brachial index; PWI: pulse } \\
\text { wave index; cPWV: central pulse wave velocity; hs-CRP: high-sensitivity C-reactive protein. Intercohortal } \\
\text { comparison was performed by defining controls as COPD GOLD } 0 \text { group, p-values refer to data } \\
\text { comparison between all five groups (COPD GOLD } 0 \text { through D). Pulmonary function parameters are based } \\
\text { on post-bronchodilator measurement. }\end{array}$} \\
\hline
\end{tabular}



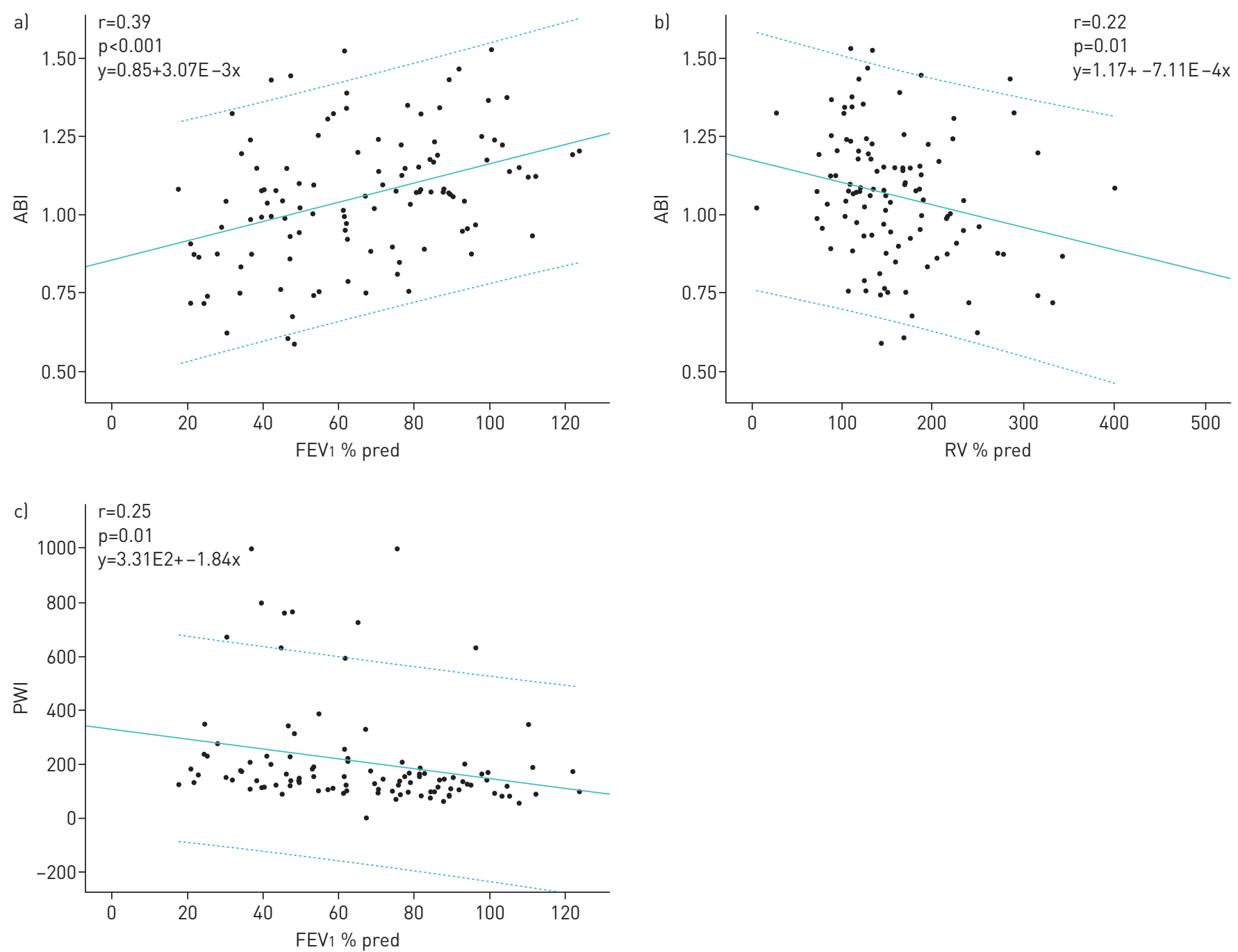

FIGURE 3 Linear regression analysis between a) forced expiratory volume in $1 \mathrm{~s}$ (FEV1) \% pred and ankle-brachial index (ABI), b) residual volume (RV) \% pred and ABI, and C) FEV1 \% pred and pulse wave index (PWI). Individual data points are presented; the solid and dashed lines indicate the mean and $95 \%$ prediction intervals, respectively.

in line with the observations made by PECCI et al. [19], WATZ et al. [20] assessed a LEAD frequency of only 25.3\% among COPD patients. These discrepant LEAD frequency data are mainly due to a different methodology: in both aforementioned studies LEAD diagnosis relied solely on ABI measurement and therein differed significantly from our angiological approach. We complemented ABI measurement by additional colour-coded duplex sonography for visualisation of the extent and severity of vascular lesions. Thereby, we found lower extremity artery plaque load to be significantly associated with the severity of obstructive airflow limitation $(\mathrm{p}<0.001)$ (table 3). Complementary pulse wave analysis permitted quantification of PWI that was likewise inversely associated with FEV1 \% pred ( $\mathrm{p}=0.01$ ) (figure $3 \mathrm{c}$ ).

When considering sole ABI measurement, the percentages of ABI values that indicate a mild (ABI 0.7 to $<0.9$ ), moderate $(\mathrm{ABI} 0.5$ to $<0.7)$ and severe peripheral circulatory compromise $(\mathrm{ABI}<0.5)$ averaged $24.3 \%, 12.1 \%$ and $5.6 \%$, respectively, within our COPD cohort. Among controls, only $13.6 \%$ exhibited an $\mathrm{ABI}$ of 0.7 to $<0.9$, whereas the remaining controls $(86.4 \%)$ presented nonpathological ABI values. These data on LEAD frequency are largely consistent with the prevalence reported by SIGVANT et al. [21]: in this Swedish population-based point-prevalence study, LEAD was diagnosed based on ABI in $18 \%$ of subjects. The fact that the final LEAD frequency among our controls increased to $54.5 \%$ supports the advantages of the presently employed additional angiological screening methods.

In the current study, we identified age to be the only cardiovascular risk factor that was independently associated with plaque presence in lower extremity arteries. Epidemiological data on LEAD show that its frequency is strongly age-related [22]. Its superiority over other common risk factors is due to the fact that 
it serves as a marker for the duration of exposure to other cardiovascular risk factors [23]. In keeping with this and on the basis of our study results, the elderly COPD patients in particular appear to be exposed to the risk of developing atherosclerotic lesions and should be directed to preventive angiological testing.

A significant relation between arterial compliance and pulmonary emphysema severity has been reported by McAllister et al. [24]. Likewise, we presently observed interdependency between the plethysmographically assessed residual volume and LEAD Fontaine stages $(\mathrm{p}=0.02)$. Thus, there is mounting evidence that suggests a persistent, largely hypoxia-mediated low-grade systemic inflammation in COPD that enhances endothelial dysfunction as a direct precursor of atherosclerosis [25]. The inflammatory cascade that initially arises from exposure to noxious substances, mainly tobacco smoke, accelerates atherogenesis at all stages by formation, destabilisation and rupture of plaque as well as by platelet activation and clotting that lead to atheroma formation and atherothrombosis $[2,26]$. In line with the assumption that systemic inflammation may link systemic vascular function to COPD status, we show that total leukocyte count is associated with both COPD and LEAD stages, and positively correlated with hs-CRP levels. hs-CRP, in turn, was significantly linked to PWI as well as to the presence and severity of carotid artery disease.

In terms of carotid artery disease, we identified carotid plaque to be significantly more prevalent in COPD patients than in control subjects, to manifest a significant increase over COPD GOLD stages and to be related to inflammation markers (leukocytes, hs-CRP). These observations are in line with the results obtained by LAHOUsSe et al. [27] who addressed the question why COPD patients are prone to ischaemic stroke and why the risk of stroke-associated mortality increases with the degree of airflow limitation. In their population-based cohort study, ultrasonography revealed a twofold increased risk of carotid artery plaque formation among COPD patients as compared with controls. Additional high-resolution carotid magnetic resonance imaging scanning showed a significantly higher presence of lipid core plaque as an indicator of plaque vulnerability. Both carotid artery wall thickening and lipid core plaque formation correlated with severity of airflow limitation [27].

There are several limitations to be considered. First, we assume that selection and procedure bias might have arisen from our single-centre, cross-sectional study design. However, we did not intend to establish causality between both disease entities, but aimed at providing an accurate estimation of the frequency of peripheral artery disease in COPD. Thus, assessment of a broader range of inflammatory biomarkers beyond total leukocyte count and hs-CRP would have been a valuable adjunct to define the linkage between systemic vascular function and COPD status. Secondly, although all COPD GOLD stages were present in our study, the number of GOLD A and C stage patients was restricted as a consequence of the study enrolment process: as patients were recruited at the University Hospital's Dept of Pneumology, less symptomatic patients (i.e. those in GOLD stages A and C) were under-represented. To overcome this limitation, we additionally combined GOLD A and B as well as GOLD C and D into two categorical variables for comparison of their respective angiological results. Moreover, we focused on FEV1 as a marker for obstructive airflow limitation rather than on COPD severity stages per se. Finally, a larger control cohort would have conferred additional comparative value to our findings. However, control enrolment was afflicted by a high number of undiagnosed COPD cases in presumably lung-healthy current or former smokers.

\section{Conclusions}

LEAD and carotid artery disease were diagnosed in an unexpectedly sizeable proportion of COPD patients as compared with control subjects. Reduced lung function and pulmonary hyperinflation were directly related to peripheral circulatory compromise. In view of the complexity of both COPD and peripheral arterial disease, the underlying pathophysiological linkage is multifactorial; yet, systemic inflammatory processes appear to be major contributing factors. These observations raise the question as to what extent effective COPD management would be advantageous with regard to onset and progress of peripheral arterial disease.

\section{References}

1 Global Initiative for Chronic Obstructive Lung Disease (GOLD). Global Strategy for the Diagnosis, Management and Prevention of COPD-2016. http://goldcopd.org/global-strategy-diagnosis-management-prevention-copd2016/ Date last updated: December 2015. Date last accessed: January 13, 2016.

2 Agusti AG. COPD, a multicomponent disease: implications for management. Respir Med 2005; 99: 670-682.

3 Agusti A, Calverley PM, Celli B, et al. Characterisation of COPD heterogeneity in the ECLIPSE cohort. Respir Res 2010; 11: 122 .

4 Fabbri LM, Luppi F, Beghé B, et al. Complex chronic comorbidities of COPD. Eur Respir J 2008; 31: 204-212.

5 McGarvey LP, John M, Anderson JA, et al. Ascertainment of cause-specific mortality in COPD: operations of the TORCH Clinical Endpoint Committee. Thorax 2007; 62: 411-415.

6 Oudijk EJ, Lammers JW, Koenderman L. Systemic inflammation in chronic obstructive pulmonary disease. Eur Respir J Suppl 2003; 46: 5s-13s.

7 Nylaende M, Kroese A, Stranden E, et al. Markers of vascular inflammation are associated with the extent of atherosclerosis assessed as angiographic score and treadmill walking distances in patients with peripheral arterial occlusive disease. Vasc Med 2006; 11: 21-28. 
8 Wozniak K, Sleszycka J, Safianowska A, et al. Systemic inflammation in peripheral arterial disease with or without coexistent chronic obstructive pulmonary disease: analysis of selected markers. Arch Med Sci 2012; 8: 477-483.

9 Tendera M, Aboyans V, Bartelink ML, et al. ESC Guidelines on the diagnosis and treatment of peripheral artery diseases: document covering atherosclerotic disease of extracranial carotid and vertebral, mesenteric, renal, upper and lower extremity arteries: the Task Force on the Diagnosis and Treatment of Peripheral Artery Diseases of the European Society of Cardiology (ESC). Eur Heart J 2011; 32: 2851-2906.

10 Arning C, Widder B, von Reutern GM, et al. Revision of DEGUM ultrasound criteria for grading internal carotid artery stenoses and transfer to NASCET measurement. Ultraschall Med 2010; 31: 251-257.

11 Hirata K, Kawakami M, O’Rourke MF. Pulse wave analysis and pulse wave velocity: a review of blood pressure interpretation 100 years after Korotkov. Circ J 2006; 70: 1231-1239.

12 Kempczinski RF. Segmental volume plethysmography in the diagnosis of lower extremity arterial occlusive disease. J Cardiovasc Surg (Torino) 1982; 23: 125-129.

13 Reiner Z, Catapano AL, De Backer G, et al. ESC/EAS Guidelines for the management of dyslipidaemias: the Task Force for the management of dyslipidaemias of the European Society of Cardiology (ESC) and the European Atherosclerosis Society (EAS). Eur Heart J 2011; 32: 1769-1818.

14 Calverley PM, Anderson JA, Celli B, et al. Salmeterol and fluticasone propionate and survival in chronic obstructive pulmonary disease. N Engl J Med 2007; 356: 775-789.

15 Anthonisen NR. Lessons from the Lung Health Study. Proc Am Thorac Soc 2004; 1: 143-145.

16 Schünemann HJ, Dorn J, Grant BJ, et al. Pulmonary function is a long-term predictor of mortality in the general population: 29-year follow-up of the Buffalo Health Study. Chest 2000; 118: 656-664.

17 Williams MC, Murchison JT, Edwards LD, et al. Coronary artery calcification is increased in patients with COPD and associated with increased morbidity and mortality. Thorax 2014; 69: 718-723.

18 Dursunoglu N, Dursunoglu D, Yildiz Aİ, et al. Severity of coronary atherosclerosis in patients with COPD. Clin Respir J 2015 [in press; DOI: 10.1111/crj.12412].

19 Pecci R, De La Fuente Aguado J, Sanjurjo Rivo AB, et al. Peripheral arterial disease in patients with chronic obstructive pulmonary disease. Int Angiol 2012; 31: 444-453.

20 Watz H, Waschki B, Boehme C, et al. Extrapulmonary effects of chronic obstructive pulmonary disease on physical activity: a cross-sectional study. Am J Respir Crit Care Med 2008; 177: 743-751.

21 Sigvant B, Wiberg-Hedman K, Bergqvist D, et al. A population-based study of peripheral arterial disease prevalence with special focus on critical limb ischemia and sex differences. J Vasc Surg 2007; 45: 1185-1191.

22 Criqui MH, Aboyans V. Epidemiology of peripheral artery disease. Circ Res 2015; 116: 1509-1526.

23 Perk J, De Backer G, Gohlke H, et al. European Guidelines on cardiovascular disease prevention in clinical practice (version 2012). Eur Heart J 2012; 33: 1635-1701.

24 McAllister DA, Maclay JD, Mills NL, et al. Arterial stiffness is independently associated with emphysema severity in patients with chronic obstructive pulmonary disease. Am J Respir Crit Care Med 2007; 176: 1208-1214.

25 Sin DD, Man SF. Systemic inflammation and mortality in chronic obstructive pulmonary disease. Can J Physiol Pharmacol 2007; 85: 141-147.

26 Maclay JD, McAllister DA, Macnee W. Cardiovascular risk in chronic obstructive pulmonary disease. Respirology 2007; 12: 634-641.

27 Lahousse L, van den Bouwhuijsen QJ, Loth DW, et al. Chronic obstructive pulmonary disease and lipid core carotid artery plaques in the elderly: the Rotterdam Study. Am J Respir Crit Care Med 2013; 187: 58-64. 